\title{
Formation Control of Nonholonomic Multi-Vehicle Systems based on Virtual Structure
}

\author{
Chika Yoshioka* Toru Namerikawa* \\ * Division of Electrical Engineering and Computer Science, \\ Graduate School of Natural Science and Technology, \\ Kanazawa University, \\ Kanazawa, JAPAN \\ toru@t.kanazawa-u.ac.jp
}

\begin{abstract}
This paper deals with formation control strategies based on Virtual Structure (VS) for multi-vehicle systems. We propose several control laws for networked multi-nonholonomic vehicle systems in order to achieve VS consensus, VS Flocking and VS Flocking with collisionavoidance.

First, Virtual Vehicle for the feedback linearization is considered, and we propose VS consensus and Flocking control laws based on a virtual structure and consensus algorithms. Then, VS Flocking control law considering collision avoidance is proposed and its asymptotical stability is proven.

Finally, simulation and experimental results show effectiveness of our proposed approaches.
\end{abstract}

\section{INTRODUCTION}

Recently there have been a lot of progress for new theories that creates a fusions of graph theories and system control theories for cooperative control problems of distributed networked control systems; e.g., Ren [2005]. A multi-agent control problem is one of significant topics where each agent works autonomously by using information of other agents over the communication network.

In the networked multi-agent systems, consensus means to reach an agreement regarding a certain quantity of interest that depends on the state of of dynamical agents. Consensus algorithm using graph theory is studied as a control problem of multi-agent systems in Olfati-Saber [2007, 2004]. Formation control problems are expected at various fields, e.g. satellites, airship, intelligent transport systems and load carriage. The consensus problems can be applied to formation control for multiple vehicles that is essential to be able to behave high-efficiency Tanner [2005, 2007], Sepulchre [2005], Ren [2006]. A vehicle is generally a nonholonomic system and it has a velocity constraint that its wheels cannot move side-away.

Many research results for formation control of nonholonomic systems have been reported Tanner [2005, 2007], Ikeda [2004]. Consensus problems with collision avoidance for multi-agent systems have been discussed in Tanner [2005, 2007], Sepulchre [2005]. However the control law could not achieve desired formation because it dose not consider control of relative position. In Ren [2006], a control law which can construct any formations, was proposed for multi-agent systems. However it has been difficult to apply it for general nonholonomic vehicle control systems. Recently there have been a lot of progress for nonholonomic formation problems e.g., in Lin [2005], Dimarogonas
[2007], but the algorithms proposed in the previous papers were complicated for real-time control applications.

On the other hand, a simple control law that makes any formation using deviation model (Virtual model) was proposed in leader-follower type, but it had no information exchange among agents in Ikeda [2004].

In this paper, we construct multi-agent systems based on virtual structure and propose novel formation control laws by using information exchange of other agents.

Several control strategies for networked multi-nonholonomic vehicle systems in order to achieve VS consensus, VS Flocking and VS Flocking with collision-avoidance are proposed. Furthermore, the asymptotical stabilities of the closed-loop system with the networked multi-nonholonomic vehicle and the proposed control strategies are proven theoretically.

Finally, the effect of the proposed control laws are evaluated via control simulations and experiments.

\section{MULTI-VEHICLE SYSTEMS}

Our controlled plants are networked multi-vehicle systems which consist $N$ vehicles ( $N$ agents) under the following assumption.

Assumption 1. There are an information network between Any $i$ th vehicle and $j$ th vehicle $(i \neq j)$ is connected and can exchange information of states of each vehicle.

Graph theory is a useful mathematical tool to represent information network structures. The network structure with Assumption 1 is said to be "connected graph" if it has bidirectional communication edges, or "strongly connected digraph" if it has unidirectional communication edges. 


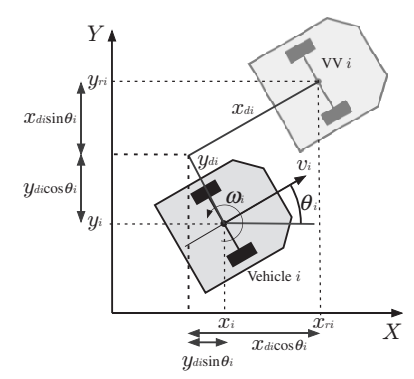

Fig. 1. $i$ th Real Vehicle and corresponding Virtual Vehicle

In this paper, we use graph Laplacian for network structures expressed mathematically. Graph Laplacian $L=\left[l_{i j}\right]$ consists of $l_{i i}=\sum_{j \neq i} a_{i j}, l_{i j}=-a_{i j}, i \neq j$ if $a_{i j}=1$ that means $j$ th vehicle send some information to $i$ th vehicle, otherwise $a_{i j}=0$.

\subsection{Vehicle Model}

The vehicle treated in this paper is a two-wheeled vehicle which is shown in Fig.1 (lower left). We assume that $N$ vehicles can be expressed via an identical model and friction force can be ignored. The kinematic model of $i$ th vehicle is described as

$$
\left[\begin{array}{c}
\dot{x}_{i} \\
\dot{y}_{i} \\
\dot{\theta}_{i}
\end{array}\right]=\left[\begin{array}{cc}
\cos \theta_{i} & 0 \\
\sin \theta_{i} & 0 \\
0 & 1
\end{array}\right]\left[\begin{array}{c}
v_{i} \\
\omega_{i}
\end{array}\right]
$$

where $\left(x_{i}, y_{i}\right)$ are the positions of center of gravity of $i$ th vehicle, $\theta_{i}$ is a heading angle of $i$ th vehicle and $v_{i}$ and $\omega_{i}$ are the control inputs. It is well known that above vehicle models have constraint on its velocity as

$$
\dot{x}_{i} \sin \theta_{i}-\dot{y}_{i} \cos \theta_{i}=0 .
$$

Therefore these vehicles are nonholonomic.

\subsection{Virtual Structure (VS)}

We consider Virtual Structure (VS) using Virtual Vehicle (VV) Ikeda [2004] for each vehicle as shown in Fig.1 (upper right). By the positional relationship between vehicle and VV in Fig.1, the kinematics model of $i$ th $\mathrm{VV}$ is described as

$$
\left[\begin{array}{c}
x_{r i} \\
y_{r i} \\
\theta_{r i}
\end{array}\right]=\left[\begin{array}{c}
x_{i}+x_{d i} \cos \theta_{i}-y_{d i} \sin \theta_{i} \\
y_{i}+x_{d i} \sin \theta_{i}+y_{d i} \cos \theta_{i} \\
\theta_{i}
\end{array}\right]
$$

where $\left(x_{r i}, y_{r i}\right)$ are positions of center of gravity of $i$ th $\mathrm{VV}, \theta_{r i}$ is heading angle of $i$ th $\mathrm{VV}$ and $x_{d i}, y_{d i}$ are distance between VVs and vehicles. The derivative of (3) are given by

$$
\begin{aligned}
& {\left[\begin{array}{c}
\dot{x}_{r i} \\
\dot{y}_{r i} \\
\dot{\theta}_{r i}
\end{array}\right]=\left[\begin{array}{c}
B_{i} \\
B_{\theta}
\end{array}\right]\left[\begin{array}{c}
v_{i} \\
\omega_{i}
\end{array}\right],} \\
& \text { where } \\
& B_{i}\left[\begin{array}{cc}
\cos \theta_{i} & -x_{d i} \sin \theta_{i}-y_{d i} \cos \theta_{i} \\
\sin \theta_{i} & x_{d i} \cos \theta_{i}-y_{d i} \sin \theta_{i}
\end{array}\right], \\
& B_{\theta}\left[\begin{array}{ll}
\Theta & 1
\end{array}\right] .
\end{aligned}
$$

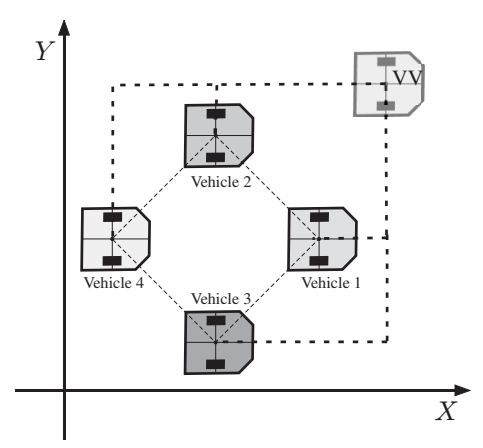

Fig. 2. Position of VSs

In this kinematics model, $B_{i}$ is nonsingular matrix if $x_{d i} \neq 0$. In this paper, we consider formation control problems for these VS systems (4).

\section{VS CONSENSUS PROBLEMS}

The goal of formation control problems is that $N$ vehicles preserve any formation based on information exchange between them over the network. To maintain any formations, the VVs of each vehicle has to converge to a common position as shown in Fig. 2 .

\subsection{Control Objectives}

To converge to a common value for $\mathrm{VV}$ of each vehicle, It is necessary to guarantee consensus for positions of center of gravity and heading angle of VVs as

$$
x_{r i} \rightarrow x_{r j}, y_{r i} \rightarrow y_{r j}, \theta_{r i} \rightarrow \theta_{r j} \quad(t \rightarrow \infty) .
$$

This consensus is called VS consensus.

Lemma 1. Consider the $N \times N$ graph Laplacian $L$ with strongly connected digraph. If the systems can be described as

$$
\dot{x}=-L_{. m} x
$$

where $x=\left[x_{1}^{T} x_{2}^{T} \cdots x_{N}^{T}\right]^{T} \in \mathbb{R}^{N m}$ are the state of all systems and $L_{. m}=L \otimes I_{m}$, the state $x$ converge as

$$
x \rightarrow\left(x_{r 1} x_{l 1}^{T} \otimes I_{m}\right) x(0)=\mathbf{1} \otimes \alpha \quad(t \rightarrow \infty),
$$

where $x_{r 1}, x_{l 1}$ are right and left eigenvector of zero eigenvalue of $L$ with $x_{l 1}^{T} x_{r 1}=1$ and $x_{l 1}^{T} \mathbf{1}=1, \otimes$ denotes Kronecker product, $\alpha \in \mathbb{R}^{m}$ is consensus value and $\mathbf{1}=$ $\left[\begin{array}{llll}1 & 1 & \cdots & 1\end{array}\right]^{T} \in \mathbb{R}^{N}$ Olfati-Saber [2004].

Proof 1. See Olfati-Saber [2004] for proof.

From Lemma 1, the all of states converge to a common value $\alpha$ as

$$
x_{1}=x_{2}=\cdots=x_{N}=\alpha
$$

\subsection{Control Law for VS Consensus}

To achieve $V S$ consensus, we propose the following control law for the vehicle $i$ as 


\section{Control law 1.}

$$
u_{i}=B_{i}^{-1}\left(-k \sum_{j \in \mathcal{N}_{i}}\left(r_{i}-r_{j}\right)+\dot{r}_{d}\right),
$$

where $u_{i}=\left[\begin{array}{ll}v_{i} & \omega_{i}\end{array}\right]^{T}, r_{i}=\left[\begin{array}{ll}x_{r i} & y_{r i}\end{array}\right]^{T}, \mathcal{N}_{i}$ is $i$ th neighbor set, $\dot{r}_{d} \in \mathbb{R}^{2}$ is constant reference velocity and $k>0$ is controller gain.

Theorem 1. Consider a system of the $N$ vehicles with kinematics (4) and Control Law 1 (10). If Assumption 1 and $\dot{r}_{d} \neq 0$ are satisfied, then $V S$ consensus achieves asymptotically.

Proof 2. All of the VS systems (4) without its angle $\theta_{r i}$ can be written as

$$
\dot{r}=\oplus \sum_{i=1}^{N} B_{i} u,
$$

where $r=\left[\begin{array}{llll}r_{1}^{T} & r_{2}^{T} & \cdots & r_{N}^{T}\end{array}\right]^{T}, u=\left[\begin{array}{llll}u_{1}^{T} & u_{2}^{T} & \cdots & u_{N}^{T}\end{array}\right]^{T}$, $\oplus \sum_{i=1}^{N} B_{i}$ is matrix that diagonal block elements are $B_{i}$. The Control law 1 (10) can be written as

$$
u=\oplus \sum_{i=1}^{N} B_{i}^{-1}\left(-k L_{.2} r+\mathbf{1} \otimes \dot{r}_{d}\right) .
$$

Let $r_{e}=r-\mathbf{1} \otimes r_{d}$, then we get the following from (11) and (12),

$$
\dot{r_{e}}=-k L_{.2} r_{e} .
$$

By Lemma 1, the systems (13) achieve consensus as $r_{e} \rightarrow$ $\mathbf{1} \otimes \alpha(t \rightarrow \infty)$. Hence, we can conclude that the positions of VVs converge to a common value as

$$
r \rightarrow \mathbf{1} \otimes\left(\alpha+r_{d}\right) \quad(t \rightarrow \infty) .
$$

The consensus for $r$ is achieved as $r_{i} \rightarrow r_{j} \rightarrow \alpha+r_{d}$. Next, we consider heading angles $\theta_{r i}$ of VVs. Substituting Control law 1 (10) into $\dot{\theta}_{r i}$ in (4) and considering $\dot{r}_{d}=$ $\left[v_{d} \cos \theta_{d} v_{d} \sin \theta_{d}\right]^{T}$, we get that

$$
\dot{\theta}_{r i}=-\frac{v_{d}}{x_{d i}} \sin \left(\theta_{r i}-\theta_{d}\right) .
$$

Hence, We have that $\theta_{r i} \rightarrow \theta_{d}(t \rightarrow \infty)$. Therefore $V S$ consensus is achieved asymptomatically. Furthermore, the any formation shape is guaranteed.

$B_{i}$ is nonsingular matrix, there is not singular value in Control Law 1 . Then, the vehicles can make any formations when VVs converge to a common value. By selecting the distance for $\operatorname{VVs}\left(x_{d i}, y_{d i}\right)$ appropriately as shown in Fig.2, the vehicles achieve any formation shapes.

The Control law 1 can be extended and the vehicles can achieve any formations even if distances for VVs are same as

$$
x_{d 1}=x_{d 2}=\cdots=x_{d N}, y_{d 1}=y_{d 2}=\cdots=y_{d N},
$$

We propose the new control law for the $i$ th vehicle as

\section{Control law 2.}

$$
u_{i}=B_{i}^{-1}\left(-k \sum_{j \in \mathcal{N}_{i}}\left(\left(r_{i}-r_{r i}\right)-\left(r_{j}-r_{r j}\right)\right)+\dot{r}_{d}\right)
$$

where $r_{r i}$ is reference relative position to $r_{i}$.

Theorem 2. Consider a system of the $N$ vehicles with kinematics (4) and Control Law 2 (17). If assumption 1 and $\dot{r}_{d} \neq 0$ are satisfied, then $V S$ consensus achieve asymptotically.

Proof 3. This can be proven in a same way with Theorem 1.

\subsection{Control Law with Velocity Tracking for VS Consensus}

The Control laws 1 and 2 include feedforward terms which are reference signals $\dot{r}_{d}$. In case of physical vehicles, the motion of vehicles are not exactly same between them. Therefore, the error of velocities $\left(\dot{r}_{d}-\dot{r}_{i}\right)$ do not converge to 0 . Consequently we propose new control law with velocity control for $i$ th vehicle as

\section{Control law 3.}

$$
\begin{aligned}
& \dot{v}_{r} \dot{\mathbf{Z}}^{*}-k_{v r}\left(v_{r i}-v^{*}\right) \\
& u B_{i}^{-1}\left(-k \sum_{j \in \mathcal{N}_{i}}\left(\left(r_{i}-r_{r i}\right)-\left(r_{j}-r_{r j}\right)\right)+v_{r i}\right)
\end{aligned}
$$

where $v^{*}$ is constant reference velocity and $k_{v r}>0$ is controller gain.

Theorem 3. Consider a system of the $N$ vehicles with kinematics (4) and Control law 3 (18). If Assumption 1 and $v^{*} \neq 0$ are satisfied, then VS consensus achieve asymptotically.

Proof 4. Substituting Control law 3 (18) into the $i$ th vehicle kinematics (4), we get that

$$
\begin{aligned}
\dot{v}_{r} & =\mathbf{1} \otimes \dot{v}^{*}-k_{v}\left(v_{r}-\mathbf{1} \otimes v^{*}\right) \\
\dot{\hat{r}} & =-k L_{.2} \hat{r}+v_{r} .
\end{aligned}
$$

Using $v_{r e}=v_{r}-\mathbf{1} \otimes v^{*}, r_{e}=\hat{r}-\int_{0}^{t} \mathbf{1} \otimes v^{*} d \tau$,

$$
\left[\begin{array}{c}
\dot{r}_{e} \\
\dot{v}_{r e}
\end{array}\right]=\left[\begin{array}{cc}
-k L_{.2} & I_{2 N} \\
0 & -k_{v r} I_{2 N}
\end{array}\right]\left[\begin{array}{c}
r_{e} \\
v_{r e}
\end{array}\right] .
$$

By Lemma 1, the systems (20) achieve consensus and velocity errors $r_{e}$ converge to 0 as

$$
r_{e} \rightarrow \mathbf{1} \otimes \alpha \quad v_{r e} \rightarrow 0
$$

Therefore any formation shape is guaranteed.

\section{VS FLOCKING PROBLEMS}

\subsection{Control Objectives}

Flocking is defined that velocity and inter-vehicle distances converge to common value. It could be as

$$
\dot{r}_{i} \rightarrow \dot{r}_{j}
$$

VS consensus problem considers only relative positions between vehicles. Here, we discuss VS Flocking problems 
that is considered both relative positions and relative velocities between VVs. The velocities is defined as $v_{r i}=$ $\left[\begin{array}{ll}v_{x i} & v_{y i}\end{array}\right]^{T}$. Then it is expressed as

$$
\dot{v}_{r i}=a_{i}, \dot{r}_{i}=v_{r i},
$$

where $a_{i}$ is control input.

\subsection{Control Law for VS Flocking}

The following control law is proposed

\section{Control law 4.}

$$
\begin{aligned}
\dot{v}_{r i} & =-\sum_{j \in \mathcal{N}_{i}} k_{i}\left(\left(\hat{r}_{i}-\hat{r}_{j}\right)+k_{v}\left(v_{r i}-v_{r j}\right)\right) \\
u_{i} & =B_{i}^{-1} v_{r i}
\end{aligned}
$$

where $k_{v}, k_{i}>0$ are controller gains.

Theorem 4. Consider a system of the $N$ vehicles with kinematics (4) and Control law 4 (18). If Assumption 1 and $1>\left|1+4 /\left(k_{v}^{2} \lambda_{i}\right)\right|$, then $V S$ Flocking achieve asymptotically, where $\lambda_{i}$ are eigenvalues of weighted graph Laplacian $L_{w}$ including $k_{i}$ and $v_{i} \rightarrow v_{j} \neq 0$.

Proof 5. The control input $\dot{v}_{r}$ for multi-vehicle systems can be written as

$$
\dot{v}_{r}=-L_{w .2} \hat{r}-k_{v} L_{w .2} v_{r} .
$$

By $B_{i}^{-1}$, the position coordinate of VS system (4) can be also described as (23). Therefore, if flocking problem achieve in second order system (23), VS systems with (4) achieve VS flocking problem. By (23) and (25), we have following result

$$
\left[\begin{array}{c}
\dot{\hat{r}} \\
\dot{v}_{r}
\end{array}\right]=\underbrace{\left[\begin{array}{cc}
0 & I_{N} \\
-L_{w} & -k_{v} L_{w}
\end{array}\right]}_{\Sigma} \otimes I_{2}\left[\begin{array}{c}
\hat{r} \\
v_{r}
\end{array}\right]
$$

$\Sigma$ has 2 zero eigenvalues. Selecting $k_{v}$ to satisfy as

$$
1>\left|1+4 /\left(k_{v}^{2} \lambda_{i}\right)\right|,
$$

where $\lambda_{i}$ is $i$ th eigenvalue of $-L_{w}$, All of eigenvalues without zero have negative real parts Ren [2006]. Finally, we consider time response of (26) and transform $\Sigma$ to $\Sigma=S J S^{-1}$ where $J$ is Jordan form composed of any vector as $S=\left[\begin{array}{llll}\omega_{1} & \omega_{2} & \cdots & \omega_{2 N}\end{array}\right], S^{-1}=\left[\begin{array}{llll}\nu_{1} & \nu_{2} & \cdots & \nu_{2 N}\end{array}\right]^{T}$. $\omega_{1}, \nu_{2}$ are right and left eigenvector of $\Sigma$ to $\lambda(\Sigma)=0$. $\omega_{2}, \nu_{1}$ are vectors that $\Sigma \omega_{2}=\omega_{1}, \nu_{1}^{T} \Sigma=\nu_{2}^{T}$. The state of multi-vehicle at $t \rightarrow \infty$ is expressed as,

$$
\begin{aligned}
{\left[\begin{array}{c}
\hat{r} \\
v_{r}
\end{array}\right] } & =\lim _{t \rightarrow \infty} S \exp (J t) S^{-1} \otimes I_{2}\left[\begin{array}{c}
\hat{r}(0) \\
v_{r}(0)
\end{array}\right] \\
& \rightarrow\left(\omega_{1} \nu_{1}^{T}+\omega_{1} \nu_{2}^{T} t+\omega_{2} \nu_{2}^{T}\right) .2\left[\begin{array}{c}
\hat{r}(0) \\
v_{r}(0)
\end{array}\right] .
\end{aligned}
$$

The each vector is written as

$$
\omega_{1}=\left[\begin{array}{l}
\mathbf{1} \\
\mathbf{0}
\end{array}\right], \omega_{2}=\left[\begin{array}{l}
\mathbf{0} \\
\mathbf{1}
\end{array}\right], \nu_{1}=\left[\begin{array}{l}
p \\
\mathbf{0}
\end{array}\right], \nu_{2}=\left[\begin{array}{l}
\mathbf{0} \\
p
\end{array}\right],
$$

where $\mathbf{0}=\left[\begin{array}{llll}0 & 0 & \cdots\end{array}\right]^{T} \in \mathbb{R}^{N}, p$ is eigenvector of $\lambda\left(-L_{w}\right)=$ 0 and $p^{T} \mathbf{1}=1$. Then, we get

$$
\left[\begin{array}{c}
\hat{r} \\
v_{r}
\end{array}\right] \rightarrow\left[\begin{array}{c}
\left(\mathbf{1} p^{T}\right)_{.2} \hat{r}(0)+\left(\mathbf{1} p^{T}\right)_{.2} v(0) t \\
\left(\mathbf{1} p^{T}\right)_{.2} v(0)
\end{array}\right]
$$

Therefore VS Flocking is achieved asymptotically.

\subsection{Control Law with Collision Avoidance for VS Flocking}

From Theorem 4, the formation shape was guaranteed in VS Flocking problem. However, in case of physical vehicles, the collision avoidance is also important problem. It is well known that artificial potential approach is effective to avoid collisionTanner [2005]. The artificial potential gives repulsive force to other vehicles if a vehicle come close to other vehicles. Here, we use following artificial potential function Tanner [2005]

$$
U_{i}=\sum_{j \in \mathcal{N}_{i}} U_{i j}, U_{i j}=\frac{d}{\left\|r_{i j}\right\|}+\log \left\|r_{i j}\right\|,
$$

where $r_{i j}=r_{i}-r_{j}$ and $d$ is controller gain. We have to select $d$ that satisfies $d>2\left(\sqrt{x_{d i}^{2}+y_{d i}^{2}}+R_{v}\right)$ where $R_{v}$ is the largest radius of the vehicles. Then we propose following control law with collision avoidance as

\section{Control law 5.}

$$
\begin{aligned}
\dot{v}_{r i} & =u_{i}^{c o}+u_{i}^{c a} \\
u_{i} & =B_{i}^{-1} v_{r i}
\end{aligned}
$$

where

$$
\begin{aligned}
u_{i}^{c o}= & \dot{v}^{*}-k_{v r}\left(v_{r i}-v^{*}\right) \\
& -\sum_{j \in \mathcal{N}_{i}} k_{i}\left(\left(\hat{r}_{i}-\hat{r}_{j}\right)+k_{v}\left(v_{r i}-v_{r j}\right)\right) \\
u_{i}^{c a}= & -\nabla_{r_{i}} U_{i}\left|\sum_{j \in \mathcal{N}_{i}} k_{i}\left(v_{r i}-v_{r j}\right)\right|
\end{aligned}
$$

where $k_{v r}, k_{v}, \quad k_{i}>0$ are controller gains. (32) is the control law to achieve consensus and (33) is the control law to achieve collision avoidance.

Theorem 5. Consider a system of the $N$ vehicles with kinematics (4) and Control law 5 (31). If Assumption 1 and assumption of the bidirectional communication for the network, and $k_{v r}+k_{v} \lambda_{2}-f_{\max }\left\|L_{w .2}\right\|>0$ are satisfied, then VS Flocking achieves asymptotically.

Where $\lambda_{2}$ is the smallest eigenvalue of $L_{w}$ without zero eigenvalue and $f_{\max }$ is the maximum potential force of and $v^{*} \neq 0$.

Proof 6. Let $v_{e}=v_{r}-\mathbf{1} \otimes v^{*}$, then the control input $\dot{v}_{e}$ for multi-vehicle systems is written as

$$
\begin{aligned}
\dot{v}_{e}= & -k_{v r} v_{e}-L_{w .2} \hat{r}-k_{v} L_{w .2} v_{e} \\
& -\oplus \sum_{i} \nabla_{r_{i}} U_{i}\left|L_{w .2} v_{e}\right|
\end{aligned}
$$

where $\oplus \sum_{i} \nabla_{r_{i}} U_{i}$ is matrix that the diagonal block element are $\nabla_{r_{i}} U_{i}$. Now, we define the function $V$ for the system as

$$
V(x)=\frac{1}{2}\left(v_{e}^{T} v_{e}+\hat{r}^{T} L_{w .2} \hat{r}\right) \geq 0 .
$$

where $x=\left[v_{e}, \hat{r}\right]^{T}$. Because of network structure of multivehicle systems with bidirectional communication can be represented undirected graph. Then we have that $L_{w .2}=$ $L_{w .2}^{T}$. The derivative of this function along trajectories of the $\dot{V}$ are given by

$$
\dot{\forall} \hat{r}^{T} L_{w .2} \dot{\hat{r}}+v_{e}^{T} \dot{v}_{e}
$$



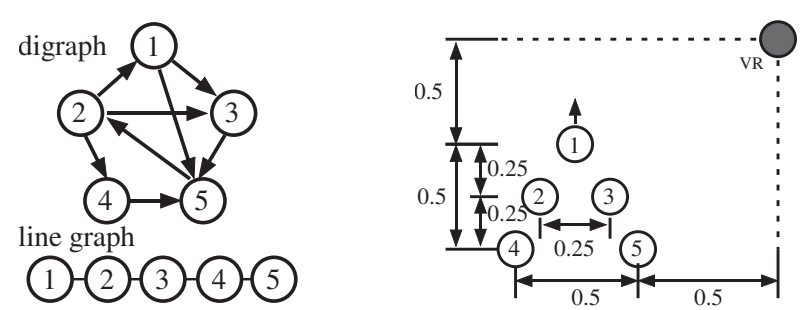

Fig. 3. Graph structure

Fig. 4. Formation

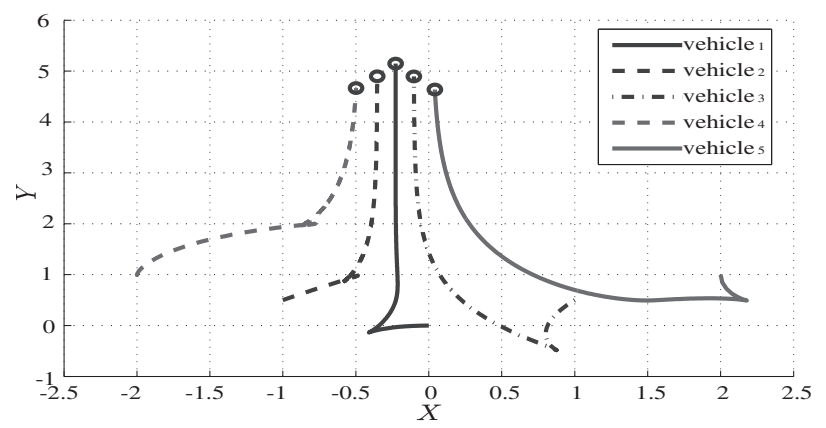

Fig. 5. Trajectory of five vehicles (VS consensus)

$$
\leq-\left(k_{v r}+k_{v} \lambda_{2}-f_{\max }\left\|L_{w .2}\right\|\right)\left\|v_{e}\right\|^{2},
$$

where $\lambda_{2}$ is smallest eigenvalue of $L_{w}$ without zero eigenvalue and $f_{\max }$ is maximum potential force. Choosing

$$
k_{v r}+k_{v} \lambda_{2}-f_{\max }\left\|L_{w .2}\right\|>0,
$$

the $\dot{V}$ is negative semi-definite. Furthermore, $\dot{V}=0$ is satisfied by only $v_{e}=0$. Applying LaSalle's invariant principle, we can see that $v_{e}$ converge to 0 asymptotically. Therefore, the consensus is achieved as $v_{r i} \rightarrow v^{*}$. Furthermore, we can see that

$$
\dot{v}_{r}=-L_{w .2} \hat{r}=0
$$

Therefore, $\hat{r}_{i} \rightarrow \hat{r}_{j}$. Thus, VS Flocking with collision avoidance is achieved asymptotically.

\section{SIMULATIONS}

Consider a group of 5 vehicles that has network structure as shown in Fig.3 (upper). Fig.4 shows the desired formation and distances of VS.

\subsection{VS Consensus Problems}

We verify the Control law 2 (10). The parameter for VS and control law are selected as $k=0.5$. The reference velocities are $\dot{r}_{d}=[0.1 \cos (\pi / 2) 0.1 \sin (\pi / 2)]^{T}$.

Fig. 5 shows the trajectory of the vehicles. From this result, the vehicles achieve desired formation and the position of $\mathrm{VVs}$ converge to a common value.

\subsection{VS Flocking Problems}

The Control law 4 (24) is examined. The parameters for VS and control law are selected as $k_{i}=0.1$ and $k_{v}=1$. The reference velocities are $\dot{r}_{d}=[0.1 \cos (\pi / 2) 0.1 \sin (\pi / 2)]^{T}$.

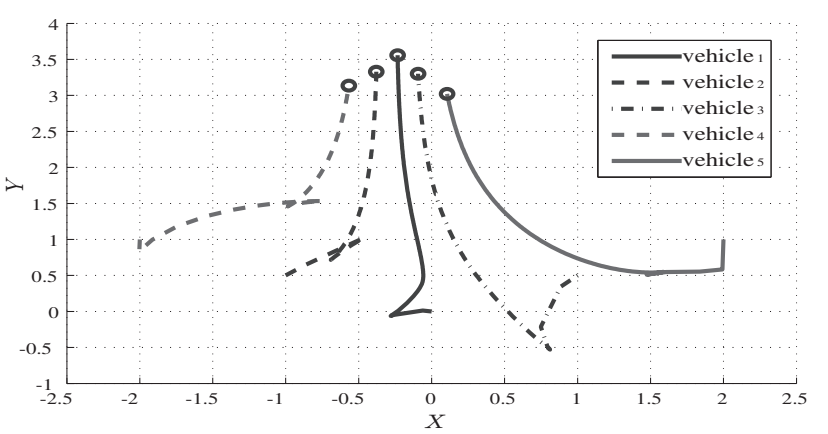

Fig. 6. Trajectory of the five vehicles (VS Flocking)
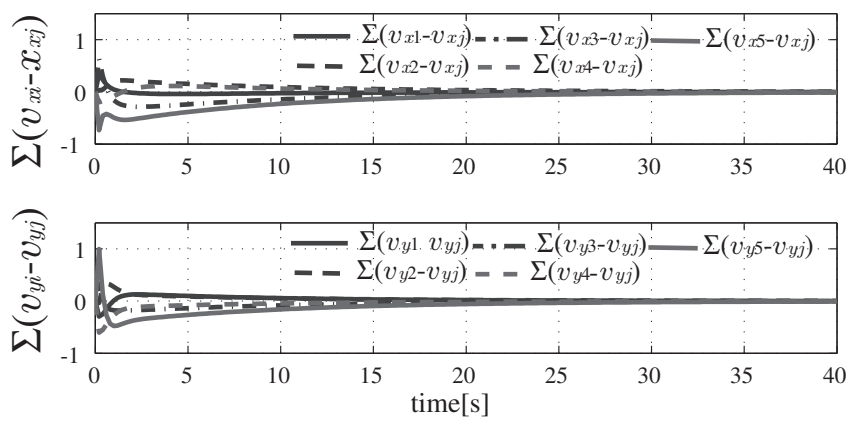

Fig. 7. Error of VSs' velocity (VS Flocking)

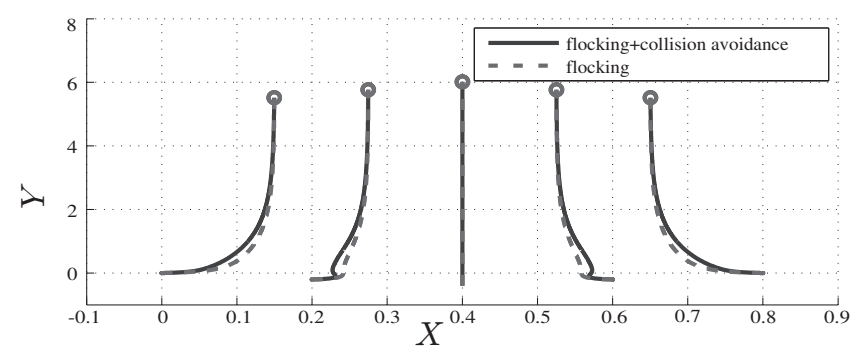

Fig. 8. Trajectory

Fig.6 shows the trajectory of the vehicles and Fig.7 shows the velocity errors between VVs. From these results, the vehicles achieve formation and the position and velocity of VVs converge to a common value.

\subsection{VS Flocking Problems with Collision Avoidance}

We verify the proposed Control law 5 (31). A group of 5 vehicles that has the network structure of line graph is considered as shown in Fig.3(lower). The parameter for VS are selected as $x_{d i}=0.05, y_{d i}=0$, i.e. the distances of $\mathrm{VVs}$ is a common value. The parameter for control law are selected as $k_{v r}=1, k_{v}=2, k_{i}=0.3$. The parameter for collision avoidance function is selected as $d=0.3$ by reason of the largest radius of the physical vehicles is $R_{v}=0.08$. The reference velocities are $v^{*}=\left[\begin{array}{ll}0.1 & \frac{\pi}{2}\end{array}\right]^{T}$. The desired formation structure is shown in Fig.4.

Fig. 8 shows simulation results in case with collision avoidance and without collision avoidance as $u_{i}^{c a}=0$. This shows that vehicles achieve formation with collision avoidance. 


\section{EXPERIMENTS}

We verify the efficacy of the proposed control laws via control experiments for $V S$ consensus problem and $V S$ Flocking problem. The experiments were carried out on 2 vehicles as shown in Fig.9. We use the dSPACE as realtime calculating machine and a sampling rate is choosen as $0.2[\mathrm{~s}]$ because of the time delay of the wireless network.

\subsection{VS Consensus Problem}

First, the proposed Control law 3 (18) for VS consensus is verified. The parameters for $\mathrm{VS}$ and control law are selected as $x_{d 1}=x_{d 2}=0.5, y_{d 1}=y_{d 2}=0, r_{r 1}=$ $\left[\begin{array}{ll}0 & 0.15\end{array}\right]^{T}, r_{r 2}=\left[\begin{array}{ll}0 & -0.15\end{array}\right]^{T}, k_{v r}=0.02, k=1$. The initial conditions are $R_{1}(0)=\left[\begin{array}{lll}0.27 & 0.18 & 0\end{array}\right]^{T}, R_{2}(0)=\left[\begin{array}{ll}0.27 & -\end{array}\right.$ $\left.\begin{array}{lll}0.18 & 0\end{array}\right]^{T}$. The reference velocity is $v_{d}=\left[\begin{array}{ll}0.07 & 0\end{array}\right]^{T}$.

Fig.10 shows the trajectory of the positions of the 2 vehicles in the field. We can see that the VVs achieve consensus.

\subsection{VS Flocking Problem}

We verify proposed Control law 5 (31) for VS Flocking problems. The parameters for VS and control law are selected as $x_{d 1}=x_{d 2}=0.1, y_{d 1}=y_{d 2}=0, r_{r 1}=$ $\left[\begin{array}{ll}0 & 0.15\end{array}\right]^{T}, r_{r 2}=[0-0.15]^{T}, k_{v r}=0.5, k_{i}=0.05, k_{v}=0.1$. The initial conditions are $R_{1}(0)=\left[\begin{array}{lll}0.3 & 0.2 & 0\end{array}\right]^{T}, R_{2}(0)=$ $\left[\begin{array}{lll}0.3 & -0.2 & 0\end{array}\right]^{T}$. The reference velocity is $v_{d}=\left[\begin{array}{lll}0.07 & 0\end{array}\right]^{T}$.

Fig.11 shows the trajectories of the positions of the vehicles in the field and this shows the VVs achieve flocking.

\section{CONCLUSIONS}

In this paper, we proposed the formation control strategies for networked multi-vehicle systems using virtual structure. Our proposed control laws could achieve desired formations for nonholonomic systems.

Several control strategies for networked multi-nonholonomic vehicle systems in order to achieve VS consensus, VS Flocking and VS Flocking with collision-avoidance were proposed.

The asymptotical stabilities of the closed-loop system with the networked multi-nonholonomic vehicle and the proposed control strategies were proven theoretically.

Finally, the effect of the proposed control laws were evaluated via control simulations and experiments which demonstrated the effectiveness of our approaches.

\section{REFERENCES}

Wei Ren, Randal W. Beard and Ella M. Atkins, "A Survey of Consensus Problems in Multi-agent Coordination," in Proc. of American Control Conference, pp. 1859-1864, June, 2005.

Reza Olfati-Saber, J.Alex Fax, Richard Murray, "Consensus and Cooperation in Networked Multi-Agent Systems," in Proc. IEEE, Vol. 95, No. 1, 2007.

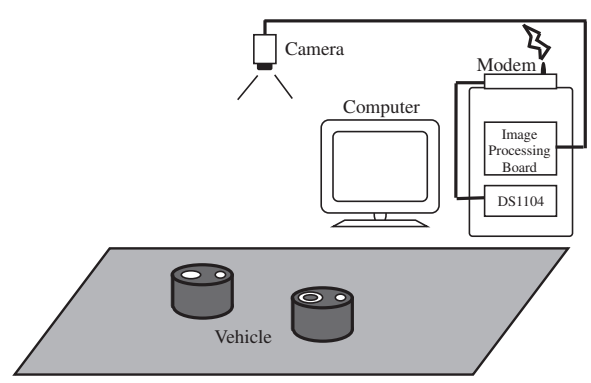

Fig. 9. Experimental setup

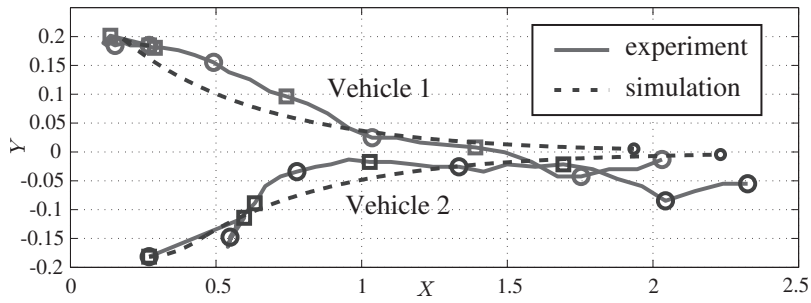

Fig. 10. Trajectory of two vehicles (VS consensus)

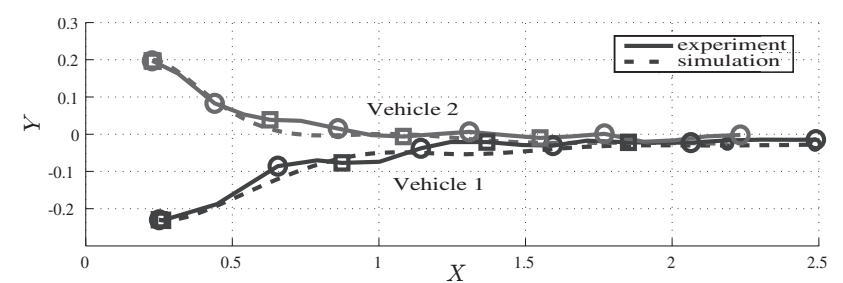

Fig. 11. Trajectory of two vehicles (VS Flocking)

Reza Olfati-Saber and Richard Murray, "Consensus Problems in Networks of Agents With Switching Topology and Time-Delays," IEEE Trans. Automatic Control, Vol. 49, No. 9, pp. 1520-1532, 2004.

Herbert G. Tanner, Ali Jadbabaie and George J. Pappas, "Flocking in Teams of Nonholonomic Agents," Cooperative Control, LNCIS 309, pp. 229-239, 2005.

Herbert G. Tanner, Ali Jadbabaie, and George J. Pappas, "Flocking in Fixed and Switching Networks," IEEE Trans. on Automatic Control, Vol.52, No. 4, 2007.

Rodolphe Sepulchre, Derek Paly and Naomi Leonard, "Collective Motion and Oscillator Synchronization," Cooperative Control, LNCIS, Vol. 309, pp. 189-205, 2005.

Wei Ren, "Consensus Based Formation Control Strategies for Multi-vehicle Systems," in Proc. of American Control Conference, pp. 4237-4242, 2006.

Takashi Ikeda, Jurachart Jongusuk, Takayuki Ikeda and Tsutomu Mita, "Formation Control of Multiple Nonholonomic Mobile Robots," IEEJ Trans. IA, Vol. 124, No. 8, pp. 814-819, 2004. (in Japanese)

Zhiyun Lin, Bruce Francis and Manfredi Maggiore, "Necessary and Sufficient Graphical Conditions for Formation Control of Unicycles," IEEE Trans. on Automatic Control, Vol. 50, No. 1, pp. 121-127, 2005.

Dimos V. Dimarogonas and Kostas J. Kyriakopoulos, "On the Rendezvous Problem for Multiple Nonholonomic Agents," IEEE Trans. on Automatic Control, Vol. 52, No. 5, pp. 916-922, 2007. 